\title{
ELEMENTARY FUNCTIONS OF A QUATERNION VARIABLE AND SOME APPLICATIONS
}

Bimurat Sagindykov, Nassibeli Jumagulova

Candidate $(\mathrm{PhD})$ of Physical and Mathematical sciences, KazNTU, Almaty, Kazakhstan

bimurat55@gmail.com

Senior Lecturer, KazNTU, Almaty, Kazakhstan

nassibeli@mail.ru

\section{ABSTRACT}

In this paper, an effective formula for the calculation of the elementary functions of a quaternion variable obtained using the methods of differential equations. Also the elementary functions are obtained from the quaternion matrices.

\section{Indexing terms/Keywords}

Quaternion algebra, an analogue of the Euler formula, matrix exponential.

\section{Academic Discipline And Sub-Disciplines}

Mathematics

\section{SUBJECT CLASSIFICATION}

Mathematics Subject Classification

\section{TYPE (METHOD/APPROACH)}

Mathematical logic

\section{Council for Innovative Research}

Peer Review Research Publishing System

Journal: Journal of Social Sciences Research

Vol .7, No.2

jssreditor.cir@gmail.com

www.jssronline.com 


\section{QUATERNION ALGEBRA}

According to Hamilton, quaternion is a mathematical object which we can write in the form [1]

$$
q \equiv q_{0}+i q_{1}+j q_{2}+k q_{3},
$$

where $q_{0}, q_{1}, q_{2}, q_{3}$ are real numbers, $q$ is called quaternion's components, the basis element « 1 » is the identity element of $q_{0}$, and $\mathrm{i}, \mathrm{j}, \mathrm{k}$ are three imaginary units. Quaternion product is denoted by «o» sign; and defined by the following rules for quaternion units multiplication given by Hamilton's definition:

$$
i \circ i=j \circ j=k \circ k=-1, i \circ j=k, j \circ k=i, k \circ i=j .
$$

We may also recognize $i, j, k$ as unit vectors $\overrightarrow{e_{1}}, \overrightarrow{e_{2}}, \overrightarrow{e_{3}}$ of Cartesian basis, and then by analogy with complex numbers, quaternion $q \in H$ can be represented as a formal sum of the scalar part $q_{0}$ and the vector part $\vec{q}$ :

$$
q=q_{0}+q_{1} \overrightarrow{e_{1}}+q_{2} \overrightarrow{e_{2}}+q_{3} \overrightarrow{e_{3}}=q_{0}+\vec{q},
$$

and the multiplication rules of basis vectors (2) can be expressed in the form of scalar and vector products:

$$
\begin{gathered}
\overrightarrow{e_{i}} \circ \overrightarrow{e_{j}}=-\left(\overrightarrow{e_{i}} \cdot \overrightarrow{e_{j}}\right)+\overrightarrow{e_{i}} \times \overrightarrow{e_{j}} \text { or } \\
\overrightarrow{e_{i}} \circ \overrightarrow{e_{j}}=-\left(\overrightarrow{e_{i}} \cdot \overrightarrow{e_{j}}\right)+\varepsilon_{i j k} \overrightarrow{e_{k}},
\end{gathered}
$$

where $\varepsilon_{i j k}$ - Levi-Civita symbols, and $i, j, k=1,2,3$.

These relationships allow us to interpret quaternions multiplication $\Lambda=\lambda_{0}+\vec{\lambda}, M=\mu_{0}+\vec{\mu}$ via scalar and vector products

$$
\Lambda \circ M=\lambda_{0} \mu_{0}-(\vec{\lambda} \cdot \vec{\mu})+\lambda_{0} \vec{\mu}+\mu_{0} \vec{\lambda}+\vec{\lambda} \times \vec{\mu} .
$$

It follows from multiplication rules for quaternion imaginary units that $q$ multiplication is non-commutative

$$
q_{1} \circ q_{2} \neq q_{2} \circ q_{1},
$$

so, there is a concept of the left and right multiplication although $q$ multiplication is still associative

$$
\left(q_{1} \circ q_{2}\right) q_{3}=q_{1}\left(q_{2} \circ q_{3}\right) \text {. }
$$

Following the procedure of obtaining conjunction we introduce the operation of quaternion conjunction

$$
q^{*} \equiv q_{0}-i q_{1}-j q_{2}-k q_{3}
$$

and define the modulus of $q$ - number

$$
|q|=\sqrt{q \circ q^{*}}=\sqrt{q_{0}^{2}+q_{1}^{2}+q_{2}^{2}+q_{3}^{2}} .
$$

\section{ANALOGUE OF EULER'S FORMULA}

We present an analogue of Euler's formula as an example of quaternion's operations. For this, let us consider quaternion's exponential function in the form:

$$
e^{q \varphi}=e^{\left(q_{0}+i q_{1}+j q_{2}+k q_{3}\right) \varphi}
$$

where $\varphi$ is a variable of the quaternion's variable.

By virtue of the fact that $q_{0}$ is a real number, it commutes with its basis unit as well as with other imaginary units:

$$
e^{\left(q_{0}+i q_{1}+j q_{2}+k q_{3}\right) \varphi}=e^{q_{0} \varphi} \cdot e^{\left(i q_{1}+j q_{2}+k q_{3}\right)} .
$$

Assume that the exponential function is given in the next form:

$$
e^{\left(i q_{1}+j q_{2}+k q_{3}\right) \varphi}=A_{0}(\varphi)+i A_{1}(\varphi)+j A_{2}(\varphi)+k A_{3}(\varphi) .
$$

Taking derivative in (6) with respect to $\varphi$ we obtain the following equation:

$$
\left(i q_{1}+j q_{2}+k q_{3}\right) e^{\left(i q_{1}+j q_{2}+k q_{3}\right) \varphi}=A_{0}^{\prime}(\varphi)+i A_{1}^{\prime}(\varphi)+j A_{2}^{\prime}(\varphi)+k A_{3}^{\prime}(\varphi) \text { or }
$$




$$
\left(i q_{1}+j q_{2}+k q_{3}\right)\left[A_{0}(\varphi)+i A_{1}(\varphi)+j A_{2}(\varphi)+k A_{3}(\varphi)\right]=A_{0}^{\prime}(\varphi)+i A_{1}^{\prime}(\varphi)+j A_{2}^{\prime}(\varphi)+k A_{3}^{\prime}(\varphi) .
$$

Hence it follows from quaternion's equality that

$$
\begin{gathered}
A_{0}^{\prime}(\varphi)=-a_{1} A_{1}(\varphi)-a_{2} A_{2}(\varphi)-a_{3} A_{3}(\varphi), \\
A_{1}^{\prime}(\varphi)=-a_{1} A_{0}(\varphi)-a_{3} A_{2}(\varphi)+a_{2} A_{3}(\varphi), \\
A_{2}^{\prime}(\varphi)=a_{2} A_{0}(\varphi)+a_{3} A_{1}(\varphi)-a_{1} A_{3}(\varphi), \\
A_{3}^{\prime}(\varphi)=a_{3} A_{0}(\varphi)+a_{1} A_{2}(\varphi)-a_{2} A_{1}(\varphi) .
\end{gathered}
$$

Having differentiated the first equation with respect to $\varphi$ variable we get

$$
\begin{gathered}
A_{0}^{\prime \prime}(\varphi)=-a_{1} A_{1}^{\prime}(\varphi)-a_{2} A_{2}^{\prime}(\varphi)-a_{3} A_{3}^{\prime}(\varphi)=-a_{1}\left[-a_{1} A_{0}(\varphi)-a_{3} A_{2}(\varphi)+a_{2} A_{3}(\varphi)\right]-a_{2}\left[a_{2} A_{0}(\varphi)+a_{3} A_{1}(\varphi)-\right. \\
a 1 A 3 \varphi-a 3 a 3 A 0 \varphi+a 1 A 2 \varphi-a 2 A 1 \varphi=-a 12-a 22-a 32 A 0 \varphi, \text { i.e. } \\
A_{0}^{\prime \prime}(\varphi)+\left(a_{1}^{2}+a_{2}^{2}+a_{3}^{2}\right) A_{0}(\varphi)=0 .
\end{gathered}
$$

We apply initial conditions for equation (8), i.e. at $\varphi=0$ :

$$
A_{0}(\varphi)=1, A_{0}^{\prime}(\varphi)=0 \text {. }
$$

After differentiating second, third and forth equations in (7) with respect to $\varphi$ variable we obtain similar equations of the type (8) for $A_{1}(\varphi), A_{2}(\varphi)$ and $A_{3}(\varphi)$ under appropriate initial conditions:

$$
\begin{aligned}
& A_{1}^{\prime \prime}(\varphi)+\left(a_{1}^{2}+a_{2}^{2}+a_{3}^{2}\right) A_{1}(\varphi)=0, \text { here at } \varphi=0, A_{1}(\varphi)=0, A_{1}^{\prime}(\varphi)=q_{1} ; \\
& A_{2}^{\prime \prime}(\varphi)+\left(a_{1}^{2}+a_{2}^{2}+a_{3}^{2}\right) A_{2}(\varphi)=0, \text { here at } \varphi=0, A_{2}(\varphi)=0, A_{2}^{\prime}(\varphi)=q_{2} ; \\
& A_{3}^{\prime \prime}(\varphi)+\left(a_{1}^{2}+a_{2}^{2}+a_{3}^{2}\right) A_{3}(\varphi)=0, \text { here at } \varphi=0, A_{3}(\varphi)=0, A_{3}^{\prime}(\varphi)=q_{3} .
\end{aligned}
$$

Solving these equations under corresponding initial conditions we have:

$$
\begin{gathered}
A_{0}(\varphi)=\cos \sqrt{q_{1}^{2}+q_{2}^{2}+q_{3}^{2}} \varphi, \\
A_{1}(\varphi)=\frac{q_{1}}{\sqrt{q_{1}^{2}+q_{2}^{2}+q_{3}^{2}}} \sin \sqrt{q_{1}^{2}+q_{2}^{2}+q_{3}^{2}} \varphi, \\
A_{2}(\varphi)=\frac{q_{2}}{\sqrt{q_{1}^{2}+q_{2}^{2}+q_{3}^{2}}} \sin \sqrt{q_{1}^{2}+q_{2}^{2}+q_{3}^{2}} \varphi, \\
A_{3}(\varphi)=\frac{q_{3}}{\sqrt{q_{1}^{2}+q_{2}^{2}+q_{3}^{2}}} \sin \sqrt{q_{1}^{2}+q_{2}^{2}+q_{3}^{2}} \varphi .
\end{gathered}
$$

In turn, an analogue of Euler's formula for quaternion can be written as

$$
e^{\left(i q_{1}+j q_{2}+k q_{3}\right) \varphi}=\cos \sqrt{q_{1}^{2}+q_{2}^{2}+q_{3}^{2}} \varphi+\frac{i q_{1}+j q_{2}+k q_{3}}{\sqrt{q_{1}^{2}+q_{2}^{2}+q_{3}^{2}}} \sin \sqrt{q_{1}^{2}+q_{2}^{2}+q_{3}^{2}} \varphi
$$

Hence if the quaternion is used as an argument of elementary function, it can be represented as a conditional complex number with a conditional imaginary unit:

$$
\begin{gathered}
q=q_{0}+\frac{i q_{1}+j q_{2}+k q_{3}}{\sqrt{q_{1}^{2}+q_{2}^{2}+q_{3}^{2}}} \sqrt{q_{1}^{2}+q_{2}^{2}+q_{3}^{2}}, \\
q=Q_{0}+I Q_{1}, \text { где } Q_{0}=q_{0}, \\
I=\frac{i q_{1}+j q_{2}+k q_{3}}{\sqrt{q_{1}^{2}+q_{2}^{2}+q_{3}^{2}}}, Q_{1}=\sqrt{q_{1}^{2}+q_{2}^{2}+q_{3}^{2}} .
\end{gathered}
$$

Here the conditioned imaginary unit has a vector meaning in which it is a unit vector directed along $\vec{I}=\operatorname{Im} q$ vector. In such notation the quaternion retains complex number's properties:

$$
\begin{gathered}
q^{2}=Q_{0}^{2}+Q_{1}^{2}=q_{0}+q_{1}^{2}+q_{2}^{2}+q_{3}^{2} . \\
q^{2}=q \circ q=q_{0}^{2}-\left(q_{1}^{2}+q_{2}^{2}+q_{3}^{2}\right)+2 q_{0}\left(i q_{1}+j q_{2}+k q_{3}\right)=Q_{0}^{2}-Q_{1}^{2}+2 I Q_{0} Q_{1}, I^{2}=-1 .
\end{gathered}
$$

Using these properties, we can find the elementary functions of a quaternion variable. For this, we 
1) replace the quaternion with a conditional complex number $q \Rightarrow Q_{0}+I Q_{1}$;

2) expand an elementary function as a function of a complex variable $Q_{0}+I Q_{1}$;

3) and after that proceed to a converse replacement $Q_{0} \Rightarrow q_{0}$,

$$
\begin{gathered}
I \Rightarrow \frac{i q_{1}+j q_{2}+k q_{3}}{\sqrt{q_{1}^{2}+q_{2}^{2}+q_{3}^{2}}}, \\
Q_{1} \Rightarrow \sqrt{q_{1}^{2}+q_{2}^{2}+q_{3}^{2}} .
\end{gathered}
$$

Some elementary functions of quaternion are written below for illustrative purposes.

$$
\begin{gathered}
\sin q=\sin \left(Q_{0}+I Q_{1}\right)=\sin Q_{0} \cos I Q_{1}+\cos Q_{0} \sin I Q_{1}=\sin Q_{0} \operatorname{ch} Q_{1}+I \cos Q_{0} \operatorname{sh} Q_{1}=\sin q_{0} \operatorname{ch} \sqrt{q_{1}^{2}+q_{2}^{2}+q_{3}^{2}}+ \\
\frac{i q_{1}+j q_{2}+k q_{3}}{\sqrt{q_{1}^{2}+q_{2}^{2}+q_{3}^{2}}} \cos q_{0} \operatorname{sh} \sqrt{q_{1}^{2}+q_{2}^{2}+q_{3}^{2}}, \\
\cos q=\cos \left(Q_{0}+I Q_{1}\right)=\cos Q_{0} \cos I Q_{1}-\sin Q_{0} \sin I Q_{1}=\cos Q_{0} \operatorname{ch} Q_{1}-I \sin Q_{0} \sin Q_{1}= \\
\cos q_{0} \operatorname{ch} \sqrt{q_{1}^{2}+q_{2}^{2}+q_{3}^{2}}-\frac{i q_{1}+j q_{2}+k q_{3}}{\sqrt{q_{1}^{2}+q_{2}^{2}+q_{3}^{2}}} \sin q_{0} \operatorname{sh} \sqrt{q_{1}^{2}+q_{2}^{2}+q_{3}^{2}} .
\end{gathered}
$$

\section{MATRIX FORM OF QUATERNION ALGEBRA}

It is interesting to present quaternion multiplication in matrix form [2]. Let

$$
\begin{aligned}
& A=a_{0}+i a_{1}+j a_{2}+k a_{3}, \\
& B=b_{0}+i b_{1}+j b_{2}+k b_{3} .
\end{aligned}
$$

Then the product of two quaternions gives third quaternion $C=A \circ B$ and the resulting quaternion components are defined by the formula (4):

$$
\begin{aligned}
& C_{0}=a_{0} b_{0}-a_{1} b_{1}-a_{2} b_{2}-a_{3} b_{3}, \\
& C_{1}=a_{1} b_{0}+a_{0} b_{1}-a_{3} b_{2}+a_{2} b_{3}, \\
& C_{2}=a_{2} b_{0}+a_{2} b_{1}+a_{0} b_{2}-a_{1} b_{3}, \\
& C_{3}=a_{3} b_{0}-a_{2} b_{1}+a_{1} b_{2}+a_{0} b_{3} .
\end{aligned}
$$

Next we assign four-dimensional $\overrightarrow{V_{A}}=\left(a_{0}, a_{1}, a_{2}, a_{3}\right)^{\mathrm{T}}$ vector to $A$ quaternion and four-dimensional $\overrightarrow{V_{B}}=\left(b_{0}, b_{1}, b_{2}, b_{3}\right)^{\mathrm{T}}$ vector to $B$ quaternion, respectively.

Then $C$ quaternion can be associated with its own four-dimensional vector defined by as follows:

$$
\overrightarrow{V_{C}}=\overrightarrow{V_{A \circ B}}=G_{1}(A) \cdot \overrightarrow{V_{B}}=G_{2}(B) \cdot \overrightarrow{V_{A}} .
$$

Matrix $G_{1}(A)$ and matrix $G_{2}(B)$ in the expression (10) equal to

$$
G_{1}(A)=\left[\begin{array}{cccc}
a_{0} & -a_{1} & -a_{2} & -a_{3} \\
a_{1} & a_{0} & -a_{3} & a_{2} \\
a_{2} & a_{3} & a_{0} & -a_{1} \\
a_{3} & -a_{2} & a_{1} & a_{0}
\end{array}\right], G_{2}(B)=\left[\begin{array}{cccc}
b_{0} & -b_{1} & -b_{2} & -b_{3} \\
b_{1} & b_{0} & b_{3} & -b_{2} \\
b_{2} & -b_{3} & b_{0} & b_{1} \\
b_{3} & b_{2} & -b_{1} & b_{0}
\end{array}\right] .
$$

For an arbitrary quaternion $Q$ matrices $G_{1}(A)$ and $G_{2}(B)$ can be represented as

$$
G_{1}(Q)=\left(\begin{array}{cc}
q_{0} & -\vec{q}^{\mathrm{T}} \\
\vec{q} & q_{0} E_{3}+K(\vec{q})
\end{array}\right), \quad G_{2}(Q)=\left(\begin{array}{cc}
q_{0} & -\vec{q}^{\mathrm{T}} \\
\vec{q} & q_{0} E_{3}-K(\vec{q})
\end{array}\right)
$$

where $E_{3}$ is 3-by-3 unity matrix,

$$
K(\vec{q})=\left(\begin{array}{ccc}
0 & -q_{3} & q_{2} \\
q_{3} & 0 & -q_{1} \\
-q_{2} & q_{1} & 0
\end{array}\right)
$$

Some properties of $K(\vec{a})$ matrix: 


$$
\begin{gathered}
K(\vec{a}) \cdot \vec{r}=\vec{a} \times \vec{r}, \\
K(\vec{a}) \cdot \vec{a}=\vec{a} \times \vec{a}=0, \\
K^{\mathrm{T}}(\vec{a})=-K(\vec{a}), \\
K(\vec{a}) K(\vec{b})=\vec{b} \times \vec{a}-\vec{a}^{\mathrm{T}} \cdot \vec{b} \cdot E_{3} .
\end{gathered}
$$

Some properties of $G_{1}$ and $G_{2}$ matrices:

$$
\begin{array}{ccc}
G_{m}\left(A^{*}\right)=G_{m}^{\mathrm{T}}(A), & G_{m}(A+B)=G_{m}(A)+G_{m}(B), & m=1,2 ; \\
G_{1}(A \circ B)=G_{1}(A) G_{2}(B), & G_{2}(A \circ B)=G_{2}(B) G_{1}(A), & \\
G_{1}(A) G_{2}(B)=G_{2}(B) G_{1}(A), & \operatorname{det} G_{m}(A)=\|A\|^{4}, & m=1,2 .
\end{array}
$$

Lemma. For any $A \neq 0$ quaternion the following equations are valid: $G_{1}\left(A^{-1}\right)=G_{1}^{-1}(A), G_{2}\left(A^{-1}\right)=G_{2}^{-1}(A)$.

Using $G_{1}$ and $G_{2}$ matrices we can easily replace equations written in quaternions to equations in matrices. In particular, for $A=B \circ C \circ D$ quaternion the matrix form will be as follows:

$$
A=G_{1}(B) \cdot G_{2}(D) \cdot \vec{C}
$$

where $\vec{C}=\left(c_{0}, c_{1}, c_{2}, c_{3}\right)^{\mathrm{T}}$ is a four-dimensional vector related to $C$ quaternion.

\section{MATRIX EXPONENTIAL}

We apply spectral decomposition of matrix function so that to find matrix exponential.

There is an isomorphism between quaternions $q=q_{0}+i q_{1}+j q_{2}+k q_{3}$ and special form 4-by-4 matrix in terms of quaternion and matrix operations.

$$
Q=\left[\begin{array}{cccc}
q_{0} & -q_{1} & -q_{2} & -q_{3} \\
q_{1} & q_{0} & -q_{3} & q_{2} \\
q_{2} & q_{3} & q_{0} & -q_{1} \\
q_{3} & -q_{2} & q_{1} & q_{0}
\end{array}\right]
$$

Let find characteristic polynomial of $Q$ quaternion matrix

$$
\operatorname{det}(Q-\lambda E)=\left[\left(q_{0}-\lambda\right)^{2}+q_{1}^{2}+q_{2}^{2}+q_{3}^{2}\right]^{2} .
$$

Then the complex values $\lambda=q_{0}+i \sqrt{q_{1}^{2}+q_{2}^{2}+q_{3}^{2}}, \bar{\lambda}=q_{0}-i \sqrt{q_{1}^{2}+q_{2}^{2}+q_{3}^{2}}$ are the eigenvalues of the quaternion $q=q_{0}+i q_{1}+j q_{2}+k q_{3}$.

The minimal polynomial of $Q$ quaternion matrix is given by

$$
\mu_{Q}(\lambda)=\frac{(-1)^{n} \operatorname{det}(Q-\lambda E)}{D_{n-1}(\lambda)}
$$

where $D_{n-1}(\lambda)$ is $(Q-\lambda E)$ characteristic matrix's the greatest common divisor of subdeterminant of order $(n-1)$.

In our case $n=4, D_{n-1}=\left(q_{0}-\lambda\right)^{2}+q_{1}^{2}+q_{2}^{2}+q_{3}^{2}$. Then

$$
\mu_{Q}(\lambda)=\left(q_{0}-\lambda\right)^{2}+q_{1}^{2}+q_{2}^{2}+q_{3}^{2}=\left(\lambda-q_{0}-i\langle q\rangle\right) \cdot\left(\lambda-q_{0}+i\langle q\rangle\right),
$$

where $\langle q\rangle=\sqrt{q_{1}^{2}+q_{2}^{2}+q_{3}^{2}}$.

Or

$$
\mu_{Q}(\lambda)=\left[\lambda-\left(q_{0}+i\left|q-q_{0}\right|\right)\right] \cdot\left[\lambda-\left(q_{0}-i\left|q-q_{0}\right|\right)\right],
$$

where $\langle q\rangle=\left|q-q_{0}\right|$. Here $i^{2}=-1$.

Then the basic formula for $f(Q)$ is as follows:

$$
f(Q)=f\left(\lambda_{1}\right) z_{11}+f\left(\lambda_{2}\right) z_{21}
$$

where $z_{11}, z_{21} Q$ matrix components, and

$$
\lambda_{1}=q_{0}+i\left|q-q_{0}\right|
$$




$$
\lambda_{2}=q_{0}-i\left|q-q_{0}\right|
$$

Substituting $\lambda-\lambda_{1}, \lambda-\lambda_{2}$ consistently in place of $f(\lambda)$ we obtain

$$
\begin{gathered}
-2 i\left|q-q_{0}\right| z_{21}=Q-\lambda_{1} E, \\
2 i\left|q-q_{0}\right| z_{11}=Q-\lambda_{2} E,
\end{gathered}
$$

where $E$ is a unity matrix.

Hence

$$
2 i\left|q-q_{0}\right| f(Q)=\left(Q-\lambda_{2} E\right) f\left(\lambda_{1}\right)-\left(Q-\lambda_{1} E\right) f\left(\lambda_{2}\right) .
$$

Let us consider some applications of this formula.

If $f(\lambda)=\frac{1}{\lambda}$, then $\frac{1}{\lambda_{1}}, \frac{1}{\lambda_{2}}$ numbers are formula's value on $Q$ matrix spectrum. Therefore, thus function is defined on $Q$ matrix spectrum.

For this reason the basic formula (11) can be used to find $Q^{-1}$ inverse matrix.

Substituting the values of $f\left(\lambda_{1}\right)=\frac{1}{\lambda_{1}}=\frac{\lambda_{2}}{\Delta}, f\left(\lambda_{2}\right)=\frac{1}{\lambda_{2}}=\frac{\lambda_{1}}{\Delta}$ into the basic formula $f(Q)$ we have $Q^{-1}=\frac{1}{\Delta}\left(-Q+2 q_{0} E\right)$. Here

$$
\Delta=q_{0}^{2}+q_{1}^{2}+q_{2}^{2}+q_{3}^{2}
$$

Validity of the obtained formula can be verified by direct calculation.

Now we find the exponent of quaternion matrix. For this, consider $f(\lambda)=e^{\lambda}$ function, which is also defined $Q$ matrix spectrum

$$
\exp (Q)=e^{q_{0}}\left[\frac{\sin \left|q-q_{0}\right|}{\left|q-q_{0}\right|} Q+\left(\cos \left|q-q_{0}\right|-\frac{q_{0} \sin \left|q-q_{0}\right|}{\left|q-q_{0}\right|} E\right)\right] .
$$

By proceeding this process we can get entire spectrum of elementary matrix of $Q$ quaternion matrix.

\section{REFERENCES}

1. Hamilton, W. R., 1994. Reasoning about naming systems. Selecta: Optics. Dynamics. Quaternions. Moscow, Nauka. Russian edition.

2. Bellman, R. 1969. Introduction to matrix analysis. Moscow, Nauka. Russian edition. 